\title{
Gilbert syndrome with systemic lupus erythematosus presenting with persistent unconjugated hyperbilirubinemia: A case report
}

\author{
NAIFANG YE $^{1 *}$, ZHUOCHAO ZHOU $^{2 *}$, HUIYUN GONG $^{3}$, \\ JIALING TENG ${ }^{2}$, YUE HAN ${ }^{4}$, CHENGDE YANG $^{2}$ and JUNNA YE ${ }^{2}$ \\ ${ }^{1}$ Department of Clinical Laboratory Medicine, The Second Affiliated Hospital of Anhui Medical University, \\ Anhui Medical University, Hefei, Anhui 230601; Departments of ${ }^{2}$ Rheumatology and Immunology, \\ ${ }^{3}$ Clinical Laboratory Medicine and ${ }^{4}$ Infectious Diseases, Ruijin Hospital, \\ Shanghai Jiao Tong University School of Medicine, Shanghai 200025, P.R. China
}

Received November 26, 2019; Accepted June 3, 2020

DOI: $10.3892 /$ etm.2020.9219

\begin{abstract}
Gilbert syndrome (GS) is a hereditary unconjugated hyperbilirubinemia that results from mutations in the bilirubin uridine diphosphate-glucuronosyltransferase (UGT1A1) gene. To the best of our knowledge, there are currently no reports that focus on patients with systemic lupus erythematosus (SLE) coexisting with GS. The present study aimed to evaluate the clinical characteristics and genotype of UGT1A1 in a Chinese patient with SLE and GS. Complete medical records and laboratory data were reviewed for a patient with SLE referred to Ruijin Hospital (Shanghai, China) for treatment between March 2016 and January 2020. Genetic analysis of the UGT1A1 gene was performed by PCR amplification and Sanger sequencing. The serum total bilirubin and unconjugated bilirubin concentrations on admission were 96.2 and $86.8 \mu \mathrm{mol} / 1$, respectively. The homozygous mutation c.1456T $>$ G (p.Y486D) in exon 5 was detected in this patient. The patient had a good response to phenobarbital orally at a dose of $30 \mathrm{mg} /$ day and a decrease in serum bilirubin was observed. Elevated unconjugated hyperbilirubinemia in SLE needs to be differentiated from other diseases, such as GS, which can be diagnosed by UGT1A1 genetic sequencing.
\end{abstract}

\section{Introduction}

Gilbert syndrome (GS) is a common autosomal dominant disorder that results in intermittent hyperbilirubinemia in

Correspondence to: Professor Junna Ye or Dr Chengde Yang, Department of Rheumatology and Immunology, Ruijin Hospital, Shanghai Jiao Tong University School of Medicine, 197 Ruijin Er Road, Shanghai 200025, P.R. China

E-mail: yjn0912@qq.com

E-mail: yangchengde@sina.com

${ }^{*}$ Contributed equally

Key words: systemic lupus erythematosus, Gilbert syndrome, genotype, hyperbilirubinemia the absence of any signs or symptoms of liver disease (1). GS usually manifests in decreased activity of the uridine diphosphate-glucuronosyltransferase (UGT1A1) gene with an incidence of $\sim 5-10 \%$ in the global population from $2018(1,2)$. UGT1A1 gene encodes a UDP-glucuronosyltransferase, which transforms small lipophilic molecules, including bilirubin into water-soluble, excretable metabolites. Several variations in the UGT1A1 gene have been described, including UGT1A1*28, UGT1A1*60 and UGT1A1*93 $(3,4)$. GS in combination with diseases, such as thalassemia, glucose-6-phosphate dehydrogenase (G6PD) deficiency, spherocytosis and acute lymphoblastic leukemia may potentiate severe hyperbilirubinemia (5-9). In addition, GS may decrease plasma oxidation and affect drug metabolism, such as irinotecan hydrochloride by decreasing the ability to conjugate drugs (10). However, to the best of our knowledge, there are currently no reports about patients with systemic lupus erythematosus (SLE) coexisting with GS.

SLE is a chronic multisystem inflammatory disease characterized by the production of various autoantibodies, such as anti-double-stranded DNA antibodies (anti-dsDNA antibodies), anti-Sm antibodies and anti-SSA/SSB antibodies. Fang et al (11) indicated that hepatic manifestation triggered by SLE itself is controversial and usually asymptomatic. This is due to the fact a variety of causes need to be differentiated, such as i) an overlap of SLE with autoimmune hepatitis (AIH) or primary biliary cirrhosis; ii) an overlap of SLE with non-autoimmune hepatopathy; iii) the existence of liver injury that only relates to SLE. And elevated liver parameters seem to be common, accounting for $25-50 \%$ patients with SLE (11); however, the etiology of hepatic damage remains unclear (12). A study by Vitek et al (13) that involved 259 patients with SLE revealed that SLE disease activity was accompanied by very low serum bilirubin levels, which were caused by severe oxidative stress. Patients with GS may be protected from the development of SLE. The present study aimed to summarize the clinical characteristics, genetic type and treatment of a patient with SLE coexisting with GS.

\section{Materials and methods}

Patient characteristics, examination and treatment. A 27-year-old Chinese female patient was referred to Ruijin 
Hospital (Shanghai, China) displaying jaundice in March 2016. The jaundice began 6 years prior to admission. The patients parents did not have jaundice, and she denied the use of potential cholestasis-inducing medication. The patient presented with a malar rash, arthritis, thrombocytopenia and decreased hemoglobin, but without kidney and nervous system involvement. The patient had no symptoms of photosensitivity, alopecia or oral ulcers. Autoantibody testing revealed that the patient had a titer of 1:100 for antinuclear antibody and that anti-Sjogren's syndrome A and B antibodies were positive as well. On this basis, the patient was diagnosed with SLE according to the 2011 classification criteria of the Systemic Lupus International Collaborating Clinics (14). The patient's laboratory findings were as follows: Hemoglobin, $80 \mathrm{~g} / 1$ (reference value: $110-150 \mathrm{~g} / \mathrm{l}$ ); white blood cell count, 9.0 $\times 10^{9} / 1$ (reference value: $4.0-10.0 \times 10^{9} / 1$ ); and platelet (PLT) count, 50.0×10 $/ 1$ (reference value: $100-300 \times 10^{9} / 1$ ). The liver function test revealed that serum levels of aspartate aminotransferase, alanine aminotransferase, alkaline phosphatase and $\gamma$-glutamyl transpeptidase were normal and total bilirubin (TB) was $91.1 \mu \mathrm{mol} / 1$ (3.4-20.5 $\mu \mathrm{mol} / \mathrm{l})$, and direct bilirubin (DB) was $12.5 \mu \mathrm{mol} / 1(0.0-6.8 \mu \mathrm{mol} / \mathrm{l})$. Therefore, the patient was characterized with persistent elevated indirect (unconjugated) bilirubin (IB). For the immunosuppressive therapy, hydroxychloroquine at a dose of $200 \mathrm{mg}$ twice/day, prednisone $40 \mathrm{mg} /$ day and cyclosporine $50 \mathrm{mg}$ twice/day orally were used. The patient was in a stable condition with regard to the SLE disease activity. However, jaundice still existed. TB, DB, erythrocyte sedimentation rate (ESR) and PLT were detected in Ruijin Hospital (Shanghai, China), consecutively. The Coombs test was negative, which excluded hemolytic anemia. Transaminases and serological tests for hepatitis B and C were negative, which excluded virus hepatitis. There was no obvious abnormality on the abdominal ultrasound or bone marrow biopsy. Informed written consent and consent for publication were obtained from the patient. This study was approved by the Ethics Committee of Ruijin Hospital (ID: 2016-62).

PCR amplification and sequencing. A total of $2 \mathrm{ml}$ patient peripheral blood was collected in a tube containing ethylenediaminetetraacetic acid (EDTA). Genomic DNA was extracted from the peripheral blood sample using the membrane-based QIAamp DNA extraction kit (Qiagen $\mathrm{GmbH}$ ) according to the manufacturer's instructions. DNA concentration and purity were measured with a spectrophotometer (NanoDrop 2000; Thermo Fisher Scientific, Inc.). Primer pairs were the same as used in a previous study (15). The promoter, exons $1-5$, adjacent intronic regions and the phenobarbital response enhancer module of the UGT1A1 gene were analyzed by polymerase chain reaction (PCR). PCR mixtures were initially denatured at $95^{\circ} \mathrm{C}$ for $5 \mathrm{~min}$, followed by 35 cycles of $30 \mathrm{sec}$ $95^{\circ} \mathrm{C}$ denaturation, $30 \mathrm{sec} 54-64^{\circ} \mathrm{C}$ annealing according to the primer pair being used and $45 \mathrm{sec}$ extension at $72^{\circ} \mathrm{C}$, with a final extension at 72 for $10 \mathrm{~min}$. PCR products $(5 \mu \mathrm{l})$ were sequenced with the Big-Dye Terminator Sequencing kit and an ABI 377 automated DNA sequencer (Applied Biosystems, Thermo Fisher Scientific Inc.).
Follow-ups. The follow-up of this patient including clinical symptoms, signs and laboratory examinations, such as ESR, PLT, liver function tests was conducted every 1 to 3 months. In addition, the SLEDAI score was evaluated each time $(16,17)$. The total follow-up period was 46 months.

\section{Results}

Laboratory data and treatment. Laboratory data were recorded consecutively and were presented in Table I. TB, IB and ESR levels were elevated at admission. Then, TB and IB levels remained stable from June 2016 to October 2016. Treatment using phenobarbital at a dose of $30 \mathrm{mg} / \mathrm{day}$ was started on November 4, 2016 until November 10, 2016.

Mutation in the UGTIAl gene. A direct sequencing analysis was conducted to identify the mutation in the UGT1A1 gene of the patient. The analysis revealed a homozygous mutation from a $\mathrm{T}$ to $\mathrm{G}$ at nucleotide position 1456 in UGT1A1 exon 5 (c.1456T $>\mathrm{G})$, resulting in the substitution of aspartate to tyrosine at position 486 of the UGT1A1 protein (p.Y486D) (Fig. 1).

Outcome and follow-up period. SLE activity indicators, such as ESR, high-sensitivity C-reactive protein and anti-dsDNA antibody remained normal during the follow-up period. SLEDAI score varied from 0 to 1 . Following phenobarbital treatment for 1 week, there was a rapid decrease in bilirubin levels (Fig. 2). TB and IB were decreased and therapy was well tolerated without any side effects. After 46 months of follow up, the patient remained stable on low-dose oral prednisone (10 mg/day) and cyclosporin (50 $\mathrm{mg}$ twice/day). The latest TB and IB values were 81.5 and $71.7 \mu \mathrm{mol} / 1$, respectively (Table I).

\section{Discussion}

GS is caused by a mutation in the UGT1A1 gene resulting in impairment of glucuronidation of unconjugated bilirubin within hepatocytes. Several studies have reported the coexistence of GS and hereditary spherocytosis, G6PD deficiency, gallstone disease and other diseases $(8,18,19)$. The reports were summarized in Table II for UGT1A1 genetic mutations and related diseases during the past 4 years. Butorac et al (7) reported the coexistence of hereditary spherocytosis and GS in a 21-month-old girl with unconjugated hyperbilirubinemia. Li et al (20) reported the combination of myeloproliferative neoplasms and the presence of the insertion mutation with the (TA)6TAA box and the missense mutation $(\mathrm{G} \rightarrow \mathrm{A})$ at $211 \mathrm{bp}$ of exon 1 in the UGT1A1 gene. Recently, over 130 genetic variants in the UGT1A1 gene were associated with GS after assessing the presence of genetic polymorphisms among different ethnicities (21). East Asian individuals had a prevalence of $\sim 2 \%$ for the genetic variants in the UGT1A1 gene, while Caucasian individuals had a prevalence of 2-10\%, and Southern Asian and Middle Eastern individuals demonstrated a significantly increased prevalence of $20 \%$ (22-24). A TA insertion mutation in the TATA box [A (TA) 7TAA] (UGT1A1*28) and c0.211 G>A (p.G71R) in exon 1 (UGT1A1*6) were common (25-27). A(TA)7TAA 
Table I. Variation of laboratory data during follow-ups for the patient with systematic lupus erythematosus and coexisting Gilbert syndrome.

\begin{tabular}{|c|c|c|c|c|c|}
\hline Time, day/month/year & $\mathrm{ESR}, \mathrm{mm} / \mathrm{h}$ & PLT, $x 10^{9} / 1$ & $\mathrm{~TB}, \mu \mathrm{mol} / 1$ & $\mathrm{DB}, \mu \mathrm{mol} / 1$ & $\mathrm{IB}, \mu \mathrm{mol} / 1$ \\
\hline $28 / 03 / 2016$ & 23 & 106 & 96.2 & 9.4 & 86.8 \\
\hline $29 / 06 / 2016$ & 9 & 108 & 87.2 & 11.4 & 75.8 \\
\hline $19 / 07 / 2016$ & 13 & 90 & 90.7 & 10.5 & 80.2 \\
\hline $10 / 08 / 2016$ & 5 & 88 & 89.5 & 12.4 & 77.1 \\
\hline $11 / 10 / 2016$ & 6 & 75 & 90.7 & 10.5 & 80.2 \\
\hline $04 / 11 / 2016$ & 8 & 54 & 77.2 & 8.7 & 68.5 \\
\hline $10 / 11 / 2016$ & 7 & 50 & 54.2 & 8.9 & 45.3 \\
\hline $29 / 11 / 2017$ & 22 & 15 & 80.2 & 9.0 & 71.2 \\
\hline $30 / 05 / 2018$ & 7 & 43 & 66.5 & 8.8 & 57.7 \\
\hline 01/03/2019 & 13 & 27 & 67.6 & 9.1 & 58.5 \\
\hline $06 / 01 / 2020$ & 12 & 32 & 81.5 & 9.8 & 71.7 \\
\hline
\end{tabular}

ESR, erythrocyte sedimentation rate; PLT, platelet; TB, total bilirubin; DB, direct bilirubin; IB, indirect bilirubin.

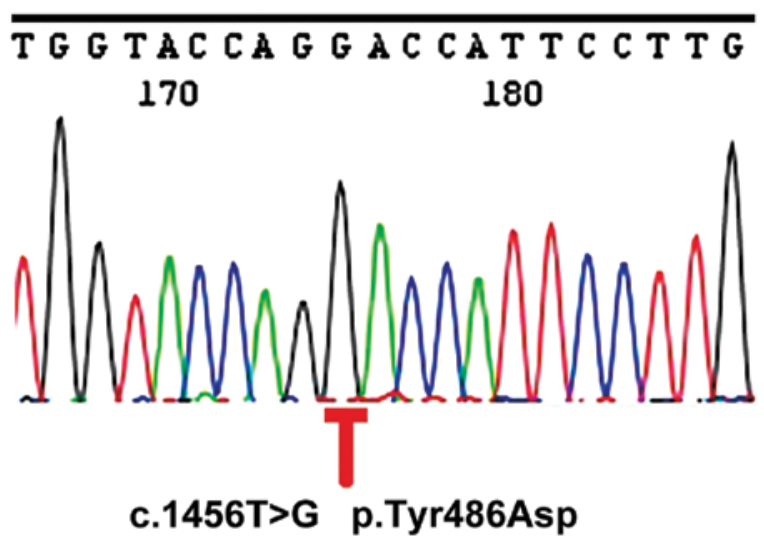

Figure 1. Mutation in the UGT1A1 gene in the patient. Sequencing analysis revealed a homozygous mutation from a $T$ to $G$ at nucleotide position 1456 in UGT1A1 exon 5 (c.1456T $>$ G), resulting in the substitution of aspartate for tyrosine at position 486 of the UGT1A1 protein (p.Y486D). Tyr, tyrosine; Asp, aspartate.

was the most common mutation in the UGT1A1 gene seen in Caucasian individuals, accounting for $\sim 35-40 \%$ (28). A study from a Romanian cohort demonstrated that the polymorphism with the highest frequency was the UGT1A1 7TA (UGT1A1*28) (29). However, a Chinese study revealed that $36.3 \%$ of patients with GS had the c.3279T $>\mathrm{G}$ mutation (30). And the frequency of A (TA) 7TAA was $30.6 \%$, which was lower compared with Caucasians (21). To the best of our knowledge, the present study demonstrated the first patient diagnosed as SLE with GS who had the homozygous mutation c.1456 T>G (p.Y486D) in the UGT1A1 gene. The patient in the present study presented with persistent unconjugated hyperbilirubinemia and had a good response to phenobarbital with a decrease in bilirubin, which confirmed the diagnosis of GS. Low-dose phenobarbital can be used continuously in patients with GS who tolerate it well. During the 46 month follow-up period, the patient exhibited stable SLE activity and serum bilirubin level. A limitation of the present study was lack of family gene verification, as the parents of the patient did not have jaundice and refused gene testing.

Nakagawa et al (31) described a single homozygote for the p.Y486D mutation in UGT1A1 exon 5, one of the shared exons, and predicted that the p.Y486D mutation may disturb the metabolization of the antipyretic and acetaminophen. This may affect the activity of the UGT1A1, UGT1A6 and UGT1A9 genes, which catalyze acetaminophen glucuronidation (32). Acetaminophen $(\sim 85 \%)$ is metabolized by conjugation, mainly glucuronidation through UDP-glucuro nosyltransferase (33). Ha et al (10) revealed that UGT1A1 genetic polymorphisms, particularly the UGT1A1*28 allele of GS may alter the metabolism of drugs, such as irinotecan hydrochloride by decreasing the ability to conjugate drugs. Attention should be paid to the use of these drugs in patients with GS.

The coexistence of SLE and GS requires further investigation. The association between serum bilirubin and SLE activity remains unclear. Serum bilirubin is the final product of hemoglobin metabolism (34). Severe hyperbilirubinemia could lead to cholestasis and neurological impairments (neurotoxicity or kernicterus), which have considerable morbidity and mortality risks (35). It was revealed that bilirubin has potent cytoprotective action due to its antiinflammatory, anti-oxidant and immunosuppressive roles at low concentrations, and mild hyperbilirubinemia prevents the development of ischemic heart disease by increasing the serum antioxidant capacity (36-39). Patients with GS had low levels of oxidative stress associated with hyperbilirubinemia (40). In addition, GS was associated with a decreased prevalence of cardiovascular disease, diabetes, endometrial cancers and with a better prognosis for Hodgkin's lymphoma $(1,39,41-43)$. In the present study, unconjugated bilirubin levels were elevated, while the SLE activity indicators remained normal and stable, such as the SLEDAI score. dos Santos et al (44) reported that unconjugated bilirubin level in SLE was negatively correlated with disease activity, which is consistent with the present study. Although the activity of SLE increases oxidative stress, serum bilirubin 
Table II. UGT1A1 genetic mutations and related diseases during the 4 year follow up period.

\begin{tabular}{|c|c|c|c|c|c|}
\hline First author, year & Number of patients & Country & Disease & Mutations & (Refs.) \\
\hline Maruo, et al 2016 & 121 & Japan & GS & $\begin{array}{l}\text { p.G71R,p.P229Q, } \\
\text { c.-3279T>G:A(TA)7TAA }\end{array}$ & (3) \\
\hline Jamwal, et al 2016 & 3 & India & GS, G6PD-deficiency, HS & $(\mathrm{TA})_{7 / 7}$ repeats & $(46)$ \\
\hline Singer, et al 2016 & 43 & Israel & GS, type I diabetes & Not mentioned & $(47)$ \\
\hline Radoi, et al 2017 & 292 & Romania & GS & $\begin{array}{l}\text { UGT1A1 (7TA), } \\
\text { UGT1A1 (8TA) }\end{array}$ & (29) \\
\hline Moyer, et al 2017 & 54 & England & GS, neonatal jaundice & $\begin{array}{l}\text { Novel variants: c.337T>G } \\
\text { (p.Y113D), c.1469A>C(p.D490A) }\end{array}$ & $(48)$ \\
\hline Li, et al 2017 & 1 & China & GS, MPN & (TA)6TAA, c.-211G>A & $(20)$ \\
\hline Sun, et al 2017 & 59 & China & GS & $\begin{array}{l}\text { c.-3279T>G, A(TA) }{ }_{7} \text { TAA, p.G71R, } \\
\text { p.P229Q, p.P364L, p.Y486D }\end{array}$ & (30) \\
\hline Aiso, et al 2017 & 1 & Japan & GS, HS & $\mathrm{A}(\mathrm{TA})_{7}$ TAA, c.211G>A:p.G71R & (49) \\
\hline Pasha, et al 2017 & 51 & Iran & GS & UGT1A1 (7TA) & $(50)$ \\
\hline Haddad, et al 2017 & 1 & Tunisia & GS, $\beta$-thalassemia & $(\mathrm{TA})_{6} /(\mathrm{TA})_{7}$ & $(51)$ \\
\hline Butorac, et al 2018 & 1 & Croatia & GS, HS & UGT1A1 (7TA) & (7) \\
\hline Qian, et al 2018 & 1 & China & GS, gallstone disease & $\begin{array}{l}\text { A(TA })_{7} \text { TAA, c. }-364 C>T \\
\text { c. }-1352 \mathrm{~A}>\mathrm{C}\end{array}$ & $(52)$ \\
\hline Bale, et al 2018 & 1,191 & India & GS, gallstone disease & UGT1A1(TA)n & (19) \\
\hline Kamal, et al 2019 & 110 & Egypt & GS & $\mathrm{A}(\mathrm{TA})_{7} \mathrm{TAA}$ & $(53)$ \\
\hline
\end{tabular}

GS, Gilbert syndrome; G6PD-deficiency, glucose-6-phosphate dehydrogenase deficiency; HS, hereditary spherocytosis; MPN, myeloproliferative neoplasm; ALL, acute lymphoblastic leukemia.

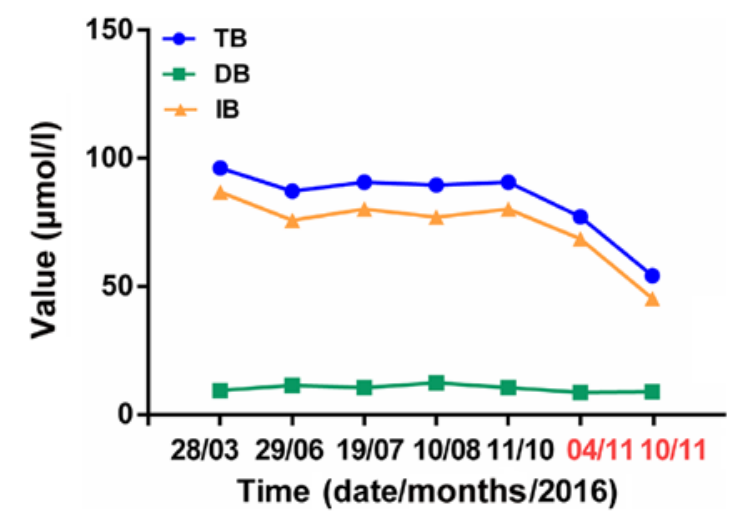

Figure 2. Variations in serum bilirubin during follow up. The figure presents the variations in serum bilirubin during follow up. The patient was treated with phenobarbital on November 4, 2016. After 1 week, there was a rapid decrease in total and indirect bilirubin levels. TB, total bilirubin; DB, direct bilirubin; IB, indirect bilirubin.

plays an important role in controlling it (45). Patients with GS can be treated with phenobarbital or no medication. When a patient experiences any other factors, such as menstruation, infection, surgery or overexertion, the degree of jaundice could be aggravated.

To conclude, the present study identified a homozygous mutation, c. $1456 \mathrm{~T}>\mathrm{G}$, in a patient with SLE with persistent hyperbilirubinemia coexisting with GS. It is pivotal that elevated unconjugated hyperbilirubinemia in SLE should be differentiated from other diseases, such as GS.

\section{Acknowledgements}

Not applicable.

\section{Funding}

The present study was supported by grants from the National Natural Science Foundation of China (grant no. 81801592), Shanghai Sailing Program (grant no. 18YF1414100), and Excellent Youth B Project (grant no. GCQN-2017-B05) and Innovative Research Team of high-level local universities in Shanghai.

\section{Availability of data and materials}

All data generated or analyzed during this study are included in this published article.

\section{Authors' contributions}

NY and ZZ drafted the manuscript. HG performed the laboratory tests. YH performed the PCR amplification and 
sequencing. JT performed the follow-up task and analysed clinical data. The experiments were designed by JY and CY. All authors read and approved the final manuscript.

\section{Ethics approval and consent to participate}

The present study was approved by the Ethics Committee of Ruijin Hospital (ID: 2016-62) and signed informed consent was obtained from the patient.

\section{Patient consent for publication}

The patient referred to in this study provided consent for the publication of her information.

\section{Competing interests}

The authors declare that they have no competing interests.

\section{References}

1. Wagner KH, Shiels RG, Lang CA, Seyed Khoei N and Bulmer AC: Diagnostic criteria and contributors to Gilbert's syndrome. Crit Rev Clin Lab Sci 55: 129-139, 2018.

2. Sieg A, Arab L, Schlierf G, Stiehl A and Kommerell B: Prevalence of Gilbert's syndrome in Germany. Dtsch Med Wochenschr 112: 1206-1208, 1987 (In German).

3. Maruo Y, Nakahara S, Yanagi T, Nomura A, Mimura Y, Matsui K, Sato H and Takeuchi Y: Genotype of UGT1A1 and phenotype correlation between Crigler-Najjar syndrome type II and Gilbert syndrome. J Gastroenterol Hepatol 31: 403-408, 2016.

4. D'Angelo R, Rinaldi C, Donato L, Nicocia G and Sidoti A: The combination of new missense mutation with [A(TA)7TAA] dinucleotide repeat in UGT1A1 gene promoter causes Gilbert's syndrome. Ann Clin Lab Sci 45: 202-205, 2015.

5. Fretzayas A, Moustaki M, Liapi O and Karpathios T: Gilbert syndrome. Eur J Pediatr 171: 11-15, 2012.

6. Berrueco R, Alonso-Saladrigues A, Martorell-Sampol L, Catala-Temprano A, Ruiz-Llobet A, Toll T, Torrebadell M, Naudo M, Camos M and Rives S: Outcome and toxicities associated to chemotherapy in children with acute lymphoblastic leukemia and Gilbert syndrome. Usefulness of UGT1A1 mutational screening. Pediatr Blood Cancer 62: 1195-1201, 2015.

7. Butorac Ahel I, Baraba Dekanic K, Palcevski G and Roganovic J: An infant with unusually high unconjugated hyperbilirubinemia due to coexistence of hereditary spherocytosis and gilbert syndrome. J Pediatr Hematol Oncol 40: e127-e128, 2018.

8. Zahedpasha Y, Ahmadpour M, Niaki HA and Alaee E: Relation between neonatal icter and gilbert syndrome in gloucose-6-phosphate dehydrogenase deficient subjects. J Clin Diagn Res 8: 63-65, 2014.

9. Tzetis M, Kanavakis E, Tsezou A, Ladis V, Pateraki E, Georgakopoulou T, Kavazarakis E, Maragoudaki E, Karpathios T and Kitsiou-Tzeli S: Gilbert syndrome associated with betathalassemia. Pediatr Hematol Oncol 18: 477-484, 2001.

10. Ha VH, Jupp J and Tsang RY: Oncology drug dosing in gilbert syndrome associated with UGT1A1: A summary of the literature. Pharmacotherapy 37: 956-972, 2017.

11. Fang X, Zaman MH, Guo X, Ding H, Xie C, Zhang X and Deng GM: Role of hepatic deposited immunoglobulin $G$ in the pathogenesis of liver damage in systemic lupus erythematosus. Front Immunol 9: 1457, 2018.

12. Chowdhary VR, Crowson CS, Poterucha JJ and Moder KG: Liver involvement in systemic lupus erythematosus: Case review of 40 patients. J Rheumatol 35: 2159-2164, 2008.

13. Vitek L, Muchova L, Jancova E, Pesickova S, Tegzova D, Peterova V, Pavelka K, Tesar V and Schwertner H: Association of systemic lupus erythematosus with low serum bilirubin levels. Scand J Rheumatol 39: 480-484, 2010.

14. Petri M, Orbai AM, Alarcon GS, Gordon C, Merrill JT, Fortin PR, Bruce IN, Isenberg D, Wallace DJ, Nived O, et al: Derivation and validation of the Systemic Lupus International Collaborating Clinics classification criteria for systemic lupus erythematosus Arthritis Rheum 64: 2677-2686, 2012.
15. Ehmer U, Kalthoff S, Fakundiny B, Pabst B, Freiberg N, Naumann R, Manns MP and Strassburg CP: Gilbert syndrome redefined: A complex genetic haplotype influences the regulation of glucuronidation. Hepatology 55: 1912-1921, 2012.

16. Bombardier C, Gladman DD, Urowitz MB, Caron D and Chang CH: Derivation of the SLEDAI: A disease activity index for lupus patients. The Committee on Prognosis Studies in SLE. Arthritis Rheum 35: 630-640, 1992.

17. Gladman DD, Ibañez D and Urowitz MB: Systemic lupus erythematosus disease activity index 2000. J Rheumatol 29: 288-291, 2002.

18. Kumar D, Parakh A and Sharma S: Gilbert syndrome increasing unconjugated hyperbilirubinemia in a child with hereditary spherocytosis. J Pediatr Hematol Oncol 34: 54-56, 2012.

19. Bale G, Avanthi US, Padaki NR, Sharma M, Duvvur NR and Vishnubhotla VRK: Incidence and risk of gallstone disease in gilbert's syndrome patients in Indian population. J Clin Exp Hepatol 8: 362-366, 2018.

20. Li XX, Shi J, Huang ZD, Shao YQ, Nie N, Zhang J, Ge ML, Huang JB and Zheng YZ: Clinical characteristics and gene mutations of gilbert syndrome complicated with myeloproliferative neoplasm. Zhongguo Shi Yan Xue Ye Xue Za Zhi 25: 567-571, 2017 (In Chinese).

21. Canu G,Minucci A,ZuppiC and Capoluongo E: Gilbert and crigler najjar syndromes: An update of the UDP-glucuronosyltransferase 1A1 (UGT1A1) gene mutation database. Blood Cells Mol Dis 50: 273-280, 2013

22. Mendez L, Lagoa M, Quiroga T, Margozzini P, Azocar L, Molina HR, Vera A, Villarroel L, Arrese M, Hampe J, et al: Prevalence of Gilbert syndrome and its genetic determinants in Chile. Rev Med Chil 141: 1266-1274, 2013 (In Spanish).

23. Gwee KA, Koay ES and Kang JY: The prevalence of isolated unconjugated hyperbilirubinaemia (Gilbert's syndrome) in subjects attending a health screening programme in Singapore. Singapore Med J 33: 588-589, 1992.

24. Hemmati F, Saki F, Saki N and Haghighat M: Gilbert syndrome in Iran, fars province. Ann Saudi Med 30: 84, 2010.

25. BosmaPJ,Chowdhury JR,Bakker C, GantlaS, deBoer A, Oostra BA, Lindhout D, Tytgat GN, Jansen PL, Oude Elferink RP, et al: The genetic basis of the reduced expression of bilirubin UDP-glucuronosyltransferase 1 in Gilbert's syndrome. N Engl J Med 333: 1171-1175, 1995.

26. Sato H, Adachi Y and Koiwai O: The genetic basis of Gilbert's syndrome. Lancet 347: 557-558, 1996.

27. Burchell B and Hume R: Molecular genetic basis of Gilbert's syndrome. J Gastroenterol Hepatol 14: 960-966, 1999.

28. Hsieh TY, Shiu TY, Huang SM, Lin HH, Lee TC, Chen PJ, Chu HC, Chang WK, Jeng KS, Lai MM and Chao YC: Molecular pathogenesis of Gilbert's syndrome: Decreased TATA-binding protein binding affinity of UGT1A1 gene promoter. Pharmacogenet Genomics 17: 229-236, 2007.

29. Radoi VE, Ursu RI, Poenaru E, Arsene C, Bohiltea CL and Bohiltea R: Frequency of the UGT1A $1 * 28$ polymorphism in a Romanian Cohort of gilbert syndrome individuals. J Gastrointestin Liver Dis 26: 25-28, 2017.

30. Sun L, Li M, Zhang L, Teng X, Chen X, Zhou X, Ma Z, Qi L and Wang P: Differences in UGT1A1 gene mutations and pathological liver changes between Chinese patients with Gilbert syndrome and Crigler-Najjar syndrome type II. Medicine (Baltimore) 96: e8620, 2017.

31. Nakagawa T, Mure T, Yusoff S, Ono E, Kusuma Harahap IS, Morikawa S, Morioka I, Takeshima Y, Nishio $\mathrm{H}$ and Matsuo M: A homozygous mutation in UGT1A1 exon 5 may be responsible for persistent hyperbilirubinemia in a Japanese girl with Gilbert's syndrome. Kobe J Med Sci 57: E26-E31, 2011.

32. Court MH, Duan SX, von Moltke LL, Greenblatt DJ, Patten CJ, Miners JO and Mackenzie PI: Interindividual variability in acetaminophen glucuronidation by human liver microsomes: Identification of relevant acetaminophen UDP-glucuronosyltransferase isoforms. J Pharmacol Exp Ther 299: 998-1006, 2001.

33. Gelotte CK, Auiler JF, Lynch JM, Temple AR and Slattery JT: Disposition of acetaminophen at 4,6, and $8 \mathrm{~g}$ /day for 3 days in healthy young adults. Clin Pharmacol Ther 81: 840-848, 2007.

34. Lester R and Schmid R: Bilirubin Metabolism. N Engl J Med 270: 779-786, 1964.

35. Watchko JF and Tiribelli C: Bilirubin-induced neurologic damage-mechanisms and management approaches. N Engl J Med 369: 2021-2030, 2013. 
36. Vitek L, Jirsa M, Brodanova M, Kalab M, Marecek Z, Danzig V, Novotny L and Kotal P: Gilbert syndrome and ischemic heart disease: A protective effect of elevated bilirubin levels. Atherosclerosis 160: 449-456, 2002.

37. Ilzecka J and Stelmasiak Z: Serum bilirubin concentration in patients with amyotrophic lateral sclerosis. Clin Neurol Neurosurg 105: 237-240, 2003.

38. Yuce S, Cure E, Cicek Y, Cumhur Cure M, Yilmaz A and Kizilkaya B: Evaluation of aortic stiffness in Gilbert syndrome patients: A protective effect of elevated bilirubin levels. Turk Kardiyol Dern Ars 43: 599-606, 2015.

39. McCarty MF: 'Iatrogenic Gilbert syndrome'-a strategy for reducing vascular and cancer risk by increasing plasma unconjugated bilirubin. Med Hypotheses 69: 974-994, 2007.

40. Maruhashi T, Soga J, Fujimura N, Idei N, Mikami S, Iwamoto Y Kajikawa M, Matsumoto T, Kihara Y, Chayama K, et al: Hyperbilirubinemia, augmentation of endothelial function, and decrease in oxidative stress in Gilbert syndrome. Circulation 126 598-603, 2012

41. Duguay Y, McGrath M, Lépine J, Gagné JF, Hankinson SE, Colditz GA, Hunter DJ, Plante M, Têtu B, Bélanger A, et al: The functional UGT1A1 promoter polymorphism decreases endometrial cancer risk. Cancer Res 64: 1202-1207, 2004.

42. Strassburg CP: Hyperbilirubinemia syndromes (GilbertMeulengracht, Crigler-Najjar, Dubin-Johnson, and Rotor syndrome). Best Pract Res Clin Gastroenterol 24: 555-571, 2010.

43. Ribrag V, Koscielny S, Casasnovas O, Cazeneuve C, Brice P Morschhauser F, Gabarre J, Stamatoullas A, Lenoir G and Salles G; Groupe d'Etude des Lymphomes agressifs group, Laboratoire de Génétique et de recherche translationnelle, and Institut Gustave Roussy: Pharmacogenetic study in Hodgkin lymphomas reveals the impact of UGT1A1 polymorphisms on patient prognosis. Blood 113: 3307-3313, 2009.

44. dos Santos BH, de R Almeida CM and Skare TL: Systemic Lupus Erythematosus activity and serum bilirubins. Acta Reumatol Port 38: 242-246, 2013.

45. Lozovoy MA, Simao AN, Panis C, Rotter MA, Reiche EM, Morimoto HK, Lavado E, Cecchini R and Dichi I: Oxidative stress is associated with liver damage, inflammatory status, and corticosteroid therapy in patients with systemic lupus erythematosus. Lupus 20: 1250-1259, 2011.
46. Jamwal M, Aggarwal A, Kumar V, Sharma P, Sachdeva MU, Bansal D, Malhotra P and Das R: Disease-modifying influences of coexistent G6PD-deficiency, Gilbert syndrome and deletional alpha thalassemia in hereditary spherocytosis: A report of three cases. Clin Chim Acta 458: 51-54, 2016.

47. Singer S, Pilpel N and Pinhas-Hamiel O: Gilbert syndrome in patients with type 1 diabetes-prevalence, glycemic control, and microalbuminuria. Pediatr Diabetes 18: 803-807, 2017.

48. Moyer AM, Skierka JM, Kotzer KE, Kluge ML, Black JL and Baudhuin LM: Clinical UGT1A1 genetic analysis in pediatric patients: Experience of a reference laboratory. Mol Diagn Ther 21: 327-335, 2017.

49. Aiso M, Yagi M, Tanaka A, Miura K, Miura R, Arizumi T, Takamori Y, Nakahara S, Maruo Y and Takikawa H: Gilbert Syndrome with concomitant hereditary spherocytosis presenting with moderate unconjugated hyperbilirubinemia. Intern Med 56: 661-664, 2017.

50. Pasha YZ, Kacho MA, Niaki HA, Tarighati M and Alaee E: The association between prolonged jaundice and TATA box dinucleotide repeats in Gilbert's syndrome. J Clin Diagn Res 11: GC05-GC07, 2017.

51. Haddad F, Trabelsi N, Chaouch L, Darragi I, Oueslati M, Boudriga I, Chaouachi D, El-Borgi W, Hafsia R, Abbes S and Ouragini H: Homozygous mutation on the $\beta$-globin polyadenylation signal in a Tunisian patient with $\beta$-Thalassemia intermedia and coinheritance of Gilbert's syndrome. Hemoglobin 41: 147-150, 2017.

52. Qian JD, Hou FQ, Wang TL, Shao C and Wang GQ: Gilbert syndrome combined with prolonged jaundice caused by contrast agent: Case report. World J Gastroenterol 24: 1486-1490, 2018.

53. Kamal S, Abdelhakam S, Ghoraba D, Massoud Y, Aziz KA, Hassan H, Hafez T and Abdel Sallam A: The frequency, clinical course, and health related quality of life in adults with Gilbert's syndrome: A longitudinal study. BMC Gastroenterol 19: 22, 2019.

c) (7) (8) This work is licensed under a Creative Commons Attribution 4.0 International (CC BY-NC 4.0) License 